\title{
A holistic approach when caring for the older adult with chronic disease and frailty
}

\begin{abstract}
Globally our population is aging fast; this is partly due to advances in health and social care services. This however is producing burden on our society and there needs to be a more integrated systems put in place to support and provide for a more structured service for the older adult. Nurses and doctors of all specialities are caring for patients with dementia in hospitals. Appropriate education and training must be available in order to deliver quality dementia care in acute hospitals.1,2 Reliable education is needed to inform hospital staff about how to care for patients who may have a superimposed diagnosis of a dementia onboard.3 However, education alone will not sustain change in practice. The need for further supervision and expert resource input is paramount to allow knowledge to enhance practice.4,5 More recent the evidence indicates that only when organisation prioritise and identify the frail older adults and perform a Comprehensive Geriatric Assessment (CGA), patient outcomes will be better. As this will allow for a treatment plan that is coordinated and person centred for the older person in the long term. 6
\end{abstract}

Volume 2 Issue 2 - 2018

\author{
Antoinette Larkin \\ Candidate Advanced Nurse Practitioner in Care of the Older \\ Adult, Portiuncula University Hospital, Ireland
}

Correspondence: Antoinette Larkin, Candidate Advanced
Nurse Practitioner in Care of the Older Adult, Portiuncula
University Hospital, Ballinasloe, Co. Galway, Ireland, Tel 090 9648245, Email Antoinette.Larkin@hse.ie

Received: February 22, 2018 | Published: April 24, 2018

\section{Introduction}

The following case report is an example of an older person living with long term conditions and severe frailty, where each diagnosis brings a new management regime of disease and an increase risk of further disability. Often signs of a cognitive impairment in the older person are firm indicators of functional disability. Four or more medications is now classed as Polypharmacy and adherence to these regimes is very much a balancing act. Recognising Delirium in the hospital is met with challenges of health care professionals not knowing the signs and symptoms. Best practice is formulated on best evidence and as health and social care professionals we must endeavour to ask the questions and take good history, so that we are able support and educate, thus empowering patients and families to self care going home.

\section{Case study}

A 77-year-old gentleman presented to the Emergency Department following acute confusion episode (Mr. D). The onset began on the previous night when Mr. D became disorientated to his home surrounding. Work up on admission included a CT Brain to out rule a stroke and Chest X-ray to out rule infection. The admitting doctor took all routine bloods-infection screen and biological-markers such as Cerium Reacting Protein (CRP) B12 Folate, Thyroid and INR levels. Blood Cultures weren't taken on regular work up due with no signs of fever and vitals recorded were within normal limits.

His previous medical history included of Pulmonary Oedema, Coronary Artery Disease (CAD) with Atrial fibrillation (A Fib) Chronic Heart Failure (CHF), following a Coronary Angioplasty and previous Stenting a year ago. Background also indicated debilitating chronic Rheumatoid Arthritis (RA), which is evident and problematic in his hands and feet. He was on a treatment plan of Methotrexate and Humira, which are both TNF antagonists. Medication analysis is a vital initial assessment and indicates bloods needed for further assessment. His treatment plan also included Fosamax 70mg for osteoporosis, supplemented with calcium tablets Calcichew 2 taken twice daily, Salazopyrin EN 500mg one tablet taken three times daily, a Proton Pump Inhibitor Protium 40mg daily. Once a week supplements included folic acid $5 \mathrm{mg}$ every Thursday. Secondary prevention included Atorvastatin $40 \mathrm{mg}$ at night, Beta-blockerBisoprolol $1.25 \mathrm{mg}$ daily, a Diuretic therapy - Frusimide 40mg once daily, and an Angiotensin Converting Enzyme (ACE) inhibitor Zestril $5 \mathrm{mg}$ daily with a blood thinner Warfarin therapy daily.

Mr. D a retired farmer lives with his wife and son in a two-story house. Family stated that he managed to take his own medication once dispensed and reported better adherence with taking his injections. His wife reported he would stop his diuretic from time to time when he was going out for the day at the fair. Mr. D had no previous history of addictions and was not taking over the counter medications, with no known drug allergies.

As a Clinical Nurse Specialist in Care of the Older Adult working collaborately within the multidisciplinary team, the two main case referrals evident in practice needing geriatric input is confusion and falls. Voyer et $\mathrm{al}^{7}$ defines episode of confusion as an onset of acute Delirium which is under diagnosed by nurses in the acute settings due to lack of knowledge to the disease process. Not recognising hypoactivity and identifying underlying dementia. On warding his wife reported recent acute Respiratory Chest Infection (RTI) accounted for a fall 2 weeks previous sustaining a facial graze and he was treated for both by his GP with an oral antibiotic. On probing his wife also reported a similar incident of confusion a few weeks previous when Mr. D was looking for a calf in a field that didn't exist, and he settled once reoriented. In wake of this new information the acting consultant commenced Co-amoxiclav a 2nd line oral antibiotic for a suspected community acquired bacterial Infection. Provision diagnosis at this early stage gave implication for a viral illness or possible unresolved RTI. Both Anti-TNF and Warfarin therapy were put on hold.

TNF Antagonists accounts for low immunity to fight infection. "Inflammatory and immunosuppressant drugs have serious adverse effects like cytotoxic drugs, they increase vulnerability to rapid spread of infection" as cited by Neal. ${ }^{8}$ Methotrexate and Humira are High 
Tech Drugs taken by subcutaneous injection weekly on different days, this combination represents a second and third line therapy where single use has not shown significant response Hoffa. ${ }^{9}$ These Anti TNF's retards disease and slows down the joint destruction and can also prevent bone resorption Kang et al. ${ }^{10}$ On its mechanism of action Methotrexate inhibits the metabolism of folic acid formed in the body from diet causes deficiency and folic acid supplement is sufficient in replacement but can also mask B12 deficiency Pietrzik et al. ${ }^{11}$ The Joint European Society of Cardiology ${ }^{12}$ identified that RA patients are at higher risks of developing CAD and vascular events, but mortality rates improves on Methotrexate treatment weekly with doses between 10-20 mg.

Given Mr. D presentation of confusion, falls and complicated drug history it was of urgency to have a diagnosis. Diagnosis is critical in developing a correct pathway of care and through proper management can provide positive outcome for both patient and carers. ${ }^{1,13}$ Under Recommendations the National Institute for Clinical Evidence Guidelines NICE ${ }^{14}$ advise under best practice to identify risks using appropriate risk calculators, treatment decisions are identified thus reducing the risk of stroke falls and CVD. Mr. D's CT brain reported normal and out ruled suspected intracranial concerns of stroke bleed / clot and his INR result was within normal clotting ratio of 3, Warfarin was recommenced. Warfarin has a narrow therapeutic index which means that it can become toxic to the system and needs close monitoring of the International Normalised Ratio (INR) in the blood. ${ }^{15}$

White Cell Count (WCC) and CRP infection marker were $<10$, which indicated a viral infection and should not be treated with Antibiotic but this does not apply to the autoimmune complicated patient's. ${ }^{16}$ In Mr. D's case, stopping the Methotrexate and Humira and starting antibiotic therapy was indicated and preformed. ${ }^{17}$ Low CRP and WCC are not of absolute significance in the setting of Methotrexate. Pre-existing and co morbidities recommend starting of antibiotic therapy NICE. ${ }^{14}$ RA patients on anti-TNF treatment should have blood cultures taken to best support treatment used, clinicians need to look for other infective signs and symptoms other than fevers.

In the U.S $75 \%$ of all antibiotic treatment is prescribed to outpatients including nonspecific RTI's and these habits are driven by patients expectations. ${ }^{18}$ Adherences to medication regimes in the elderly were compliant if normal daily routines weren't upset, and $50 \%$ need assistance with medications Sanders et al. ${ }^{19}$ The Joint European Society of Cardiology ${ }^{12}$ also acknowledged that social economical factors and complicated medication regimes were factors that influenced adherence. Lingford-Hughes ${ }^{20}$ identified that the evidence capture in addiction and misuse of prescription medication in the elderly is scarce; this is due to the poor history taking in practice.

Currently in the U.K over $40 \%$ of all patients over 75 years of age admitted to an acute hospital have a diagnosis of dementia and only half of these admitted have had a diagnosis Department of Health in UK. ${ }^{21}$ Falls in confused older patients accounts for morbidity and mortality and these risks are levied on the severity of the cognitive impairment. ${ }^{22}$ Falls in the elderly are markers of frailty and this has clinical significance if caught and acted on timely with exercise plans. ${ }^{22}$ Screening for sensory impairments should be performed in all patients in order to prevent increased delirium with increasing cognitive impairment. ${ }^{7}$ Regular vision checks are not evident for improving risk of falls NICE. ${ }^{14}$ The confusion persisted for a further 3 days, and on Saturday morning (day 4) Mr. D decided to go for a walk down town to the local Mart. He was found and returned safely to the ward where he was supervised closely until family could arrive. Family took Mr. D home with agreement from the on call medical team and a junior doctor completed a prescription, which only included an oral antibiotic for a further 4 days. The hospital setting is more detrimental to the frail elderly with acute confusion and functional decline accounting to risks of falls including adverse drug events Page et al. ${ }^{24}$ Acute confusion would be better managed in the person's own home in an ideal world. There have been a number of models of care mentioned throughout the research and dementia can be described as a loss of "self" once the person is seen as confused it is difficult to see anything else Spector et al. ${ }^{25}$ Attitudes and experience encourage isolation that threatens personhood; ways to reduce the stigma in dementia is providing direct care, Alzheimer's Society of Ireland. ${ }^{26}$ The following Monday morning the wife of Mr. D spoke with his relevant team reporting that she was finding it hard to get Mr. D to take his regular medication. Mr. D was reviewed in the Out Patients Department later that day and questioned on his medications. It transpired that the oral antibiotic was causing him to feel nauseated including loss of appetite, which meant he felt too unwell to take his tablets. Nursing records and drug kardex filed showed that he had complained of these bizarre, severe effects of feeling nauseated soon after starting the antibiotic and was commenced on an anti-emetic metoclopramide $10 \mathrm{mg} 8$ hourly by a doctor on call. This explained the masking of the Adverse Drug Reaction (ADR) in hospital. Medication mismanagement accounts ADR's falling disproportionately on the most vulnerable, the elderly with poor mental health and which $72 \%$ of these are avoidable. ${ }^{27}$

The causative routes lying with Nurse Medication routines, these are not based on the holistic assessment requirement but are focused on task-orientated needs. ${ }^{28}$ Nurses need to embrace and use prescribing to their caring advantage, taking an active control of what is prescribed using best practice and only prescribe when needed and not being influenced by their perceptions Page et al. ${ }^{24}$ Attitudes needs to change to reporting ADR's and that by only challenging the fears of disciplinary action and admitting to wrongs will nursing find its scope to practice safely. ${ }^{29}$

Age related changes in the older person are accountable for ADR's and up to $17 \%$ of all hospital patients over the age of 75 experience them Beckwith et al. ${ }^{30}$ Polypharmacy is reported in $79 \%$ of elderly patients who have a medical card on the drug payment scheme. ${ }^{31}$ The older people taking multiple drugs for multiple chronic diseases are more likely to experience ADR's. ${ }^{9}$ In Mr. D case it possibly was a non - immunological Adverse Reaction often associated with nausea and diarrhoea ${ }^{32}$ this is due to his suppressed immune system. Gould et al. ${ }^{33}$ identified older people and the immune compromised are at greater risk of RTI's in the community. The facts that ADR's are preventable; causing a costly burden increasing the lengths of stays in hospital should be better-managed using prescribing access and reporting using a clinical support database. ${ }^{34}$ This would support and flag the risks of Drug on Drug interactions when prescribing. The two biggest causes of ADR's in the Older Person are Drug on Drug and Drug on Disease. ${ }^{35}$

The acting consultant stopped the offending antibiotic, and rationalized medications to ensure continued therapy. His bone therapy was changed from once weekly to parental subcutaneous Denosumab $60 \mathrm{mg}$ taken twice a year, at which Mr. D was happy to give and organized through his local pharmacy. Choice of osteoporosis therapy should reflect and consider patient need and requirement using the 
World Health Organizational FRAX score to guide prescribing. ${ }^{36}$ His Vitamin D blood levels report indicated low serum levels with deficiency with levels $<30 \mathrm{nmol} / \mathrm{L}$ so his Calcichew tablets were changed to Calcichew D3, which increases the absorption of Calcium. Low levels of Vitamin D are lower in the elderly due to reduced capacity to synthesise on the aging skin, and increased immobility causing - reduced balance control and impaired cognition. ${ }^{37}$ Higher levels Vitamin D levels have shown to have clinical significant in fighting viral illnesses. ${ }^{38} \mathrm{~A}$ referral was made to an on-site to a pharmacist to support education and warn of ADR's thus ensuring compliance with medication regime and thereby reducing the risks of readmission..$^{39}$

Follow up referral to Psychiatry of Later Life (POLL) was sent and a MRI Brain was ordered by the POLL consultant confirmed a diagnosis of multi-infracts (mini clots/strokes), also known as Vascular Dementia (VaD). On review 2 weeks later Mr. D had returned to his normal cognitive level. A repeat Mini Mental State Exam (MMSE) ${ }^{40}$ is a 30-point questionnaire universal scale to check memory impairment indicated marked improvement. A score of 18 indicating moderate cognitive impairment, where previous score on initial assessment was 7 indicating severe cognitive impairment when $<10$. Once a diagnosis had been made a predicted cause needs to be identified in order to maximize treatment, and the use of the MMSE is a vital tool to assess levels of cognitive impairment Holmes. ${ }^{41}$ Health professionals prescribing need to follow best practice NICE Guidelines as these provide the cornerstones of safe practice and clinical governance. ${ }^{42}$ Under the NICE Guideline, patients with MMSE above the score of 10 should continue to be prescribed treatment-such as anticholinesterase inhibitors and the decisions around medication need to only be made by the Geriatric Physicians managing the patients care. ${ }^{43}$ POLL prescribed Rivastigmine 4.6mg an Acetyl Cholinesterase Inhibitor patch applied every 24 hours. Mr. D's wife was also warned on applying the new patch and advised to use upper chest and upper back only, to ensure maximum bioavailability. ${ }^{17}$

In conclusion, patient safety is paramount and through effective teamwork and communication the nurse prescriber has increased safety by providing access, satisfaction and ensuring effective selfmanagement of their own medications. ${ }^{43}$ This supports providing Mr $\mathrm{D}$ with changing the method and times of medications administration to support adherence. Medication arrangement should be identified with daily routine and lifestyle patterns assessing patients individually. Prescribing in the older adult is challenging and has no easy answers, there needs to be effective communication channels between patient and multidisciplinary both community and acute settings. Single patient information is what is recommended by NICE. ${ }^{14}$

\section{Acknowledgements}

To all the nurses and doctors who care for the older adults of Portiuncula University Hospital, Ballinasloe, Co. Galway, Ireland.

\section{Conflict of interest}

The author declares no conflict of interest.

\section{References}

1. Cahill S, O'Shea E, Pierce M. Creating excellence in dementia care: a research review for Ireland's national dementia strategy. Ireland: Springer; 2012. p. $1-52$
2. Eustace A, Bruce I, Coen R, et al. Behaviour disturbance triggers recognition of dementia by family informants. Int $J$ Geriatr Psychiatry. 2007;22(6):574-579.

3. Alzheimer's Society UK. Counting the cost: Caring for people with dementia on hospital wards. London: Springer; 2009.

4. Elvish R, Burrow S, Cawley R, et al. Getting to Know Me': the development and evaluation of a training programme for enhancing skills in care of people with dementia in general hospital settings. Aging Mental Health. 2014;18(4):481-488.

5. Royal college of psychiatrists. Who cares wins. Improving the outcome for older people admitted to the general hospital. Guidelines for the development of liaison mental health services for older people. NHS, England: Springer; 2005.

6. Robertson H. Think Delirium. NHS, Scotland: Healthcare improvement; 2014. p. $1-16$

7. Voyer P, Cole MG, Mc Cusker J. et al. Characteristics of institutionalized older patients with delirium newly admitted to an acute care hospital. Clinical Effectiveness in Nursing. 2005;9(1):13-25.

8. Neal MJ. Medical pharmacology at a glance, Fifth Edition. Reprint, Garsington Rd, Oxford: Blackwell Publishing Ltd; 2005.

9. Hoffa GW. Investigating infection with patients with RA. Clinical Reviews. 2010;20(10):38-42.

10. Kang KY, Ju JH, Park SH, et al. The paradoxical effects of TNF inhibitors on bone mineral density and radiographic progression in patients with ankylosing spondylitis. Rheumatology (Oxford). 2013;52(4):718-726.

11. Pietrzik K, Bailey L. Barry S. Folic Acid and L-5-Methyltetrahydroxfolate, comparison of clinical pharmacokinetics and pharmacodynamics. Clinical Pharmacokinetics. 2010;49(8):535-548.

12. The Joint European society of cardiology. European guidelines on cardiovascular disease prevention in clinical practice. European Heart Journal. 2012;33(13):1635-1701.

13. Thijs RD, Bloem BR, Van Dijk JG. Falls, faints, fits and funny turns. $J$ Neurol. 2009;256(2):155-167.

14. National institute for health and clinical excellence. Respiratory tract infections â "antibiotic prescribing Prescribing; 2008.

15. Lanshear A, Harden J, Simms J. Safe practice for patients receiving anticoagulant therapy. Nurs Stand. 2010;24(20):47-55.

16. Kavanagh KE, Shea OE, Halloran R, et al. A pilot study of the use of nearpatient C-reactive protein testing in the treatment of adult respiratory tract infections in one Irish general practice. BMC Fam Pract. 2011;12:93.

17. WWW.Medicines.ie

18. Leemann-Price E, MacKenzie TD, Metlay JP, et al. A computerized education module improves patient knowledge and attitudes about appropriate antibiotic use for acute respiratory tract infections. Patient Educ Couns. 2011;85(3):493-498.

19. Sander M, Van Oss T. Using daily routines to promote medication adherence in the older adults. Am J Occup Ther. 2013;67(1):91-99.

20. Lingford-Hughes AR, Welch S, Peters L, et al. BAP updated guidelines: evidence-based guidelines for the pharmacological management of substance abuse, harmful use, addiction and comorbidity: recommendations from BAP. J Psychopharmacol. 2012;26(7):899-952.

21. Department of health UK. Commitment to the care of people with dementia in hospital settings. royal college of nursing, UK: Springer; 2012. p. 1-30. 
22. Tzeng H. Inpatient falls in adult acute care settings: influence of patients mental status. $J$ Adv Nurs. 2010;66(8):1741-1746.

23. Ahmed N, Mandel R, Mindy J. Frailty: an emerging geriatric syndrome. Am J Med. 2007;120(9):748-753.

24. Page RL, Linnebur SA, Bryant LL. et al. Inappropriate prescribing in the hospitalized elderly patient: Defining the problem, evaluation tools, and possible solutions. Clin Inter Aging. 2010;5:775-787.

25. Spector A, Orrell M. Using a biopsychosocial model of dementia as a tool to guide clinical practice. Int Psychoger. 2010;22(6):957-965.

26. The Alzheimer's society of Ireland. Preceptions of stigma in dementia: an exploratory study. The school of nursing and midwifery Trinity College Dublin. 2006. p. 1-116.

27. Jordan S. Adverse Drug Reactions: reducing the burden of Treatment. Nursing Standard. 2007;21(34):35-41.

28. Bolster D, Manias E. Person-centered interactions between nurses and patients during medication activities in an acute hospital setting: Qualitative observation and interview. Int J Nurs Stud. 2010;47(2):154-165.

29. Boyle D, Schulmeister A, Lajeunesse L, et al. Medication misadventure in cancer care. Seminars in Oncol Nur. 2002;18(2):109-120.

30. Beckwith S, Frankin. Oxford handbook of nurse prescribing. Br J Clin Pharmacol. 2007;64(1):115.

31. Richardson K, Moore P, Peklar J, et al. Polypharmacy in adults over 50 in Ireland: Opportunities for cost saving and improved healthcare. Tilda. 2012. p. 1-37.

32. Hall C, Allen J, Barlow G. Antibiotic prophylaxis. Surgery (Oxford). 2012;30(12):651-658.

33. Gould D, Drey N. Preventing the spread of acute respiratory viral infection. Nurs Stand. 2009;24(5):44-49.
34. Wright A, FebloWitz F, PhAnsAlkAr S. et al. Preventability of adverse drug events involving multiple drugs using publicly available clinical decision support tools. Am J Health Syst Pharm. 2012;69(3):221-227.

35. O’Connor MN, Gallagher P, Byrne S, et al. Adverse drug reactions in older patients during hospitalisation: are they predictable? Age Aging. 2012;41(6):771-776.

36. Silverman S, Christiansen C. Individualizing osteoporosis therapy. Osteoporosis International. 2012;23(3):797-809.

37. Menant JC, Close JC, Delbaere K, et al. Relationships between serum vitamin levels, neuromuscular and neuropsychological function and falls in older men and women. Osteoporos Int. 2012;23(3):981-989.

38. Sebetta JR, DePetrillo P, Cipriani RJ, et al. Serum 25-hydroxyvitamin $\mathrm{d}$ and the incidence of acute viral respiratory tract infections in healthy adults. PloS One. 2010;5(6):e11088.

39. Donihi AC, Weber RJ, Sirio CA, et al. An Advanced Pharmacy Practice Experience in Inpatient Medication Education. Am J Pharm Educ. 2009;73(1):11.

40. Folstein MF, Folstein SE, McHugh PR. Mini-mental state' A practical method for grading the cognitive state of patients for the clinician. $J$ Psychiatr Res. 1975;12(3):189-198.

41. Holmes C. Dementia. Plum X Matrix. 2012;40(11):628-631.

42. Walsh K, Sanders J, Susheel SK. et al. Getting NICE guidelines into practice: can e-learning help? Journal of Clinical Governance. 2010;15(1):6-11.

43. Mathews F, Muniz-Terrera G, McKeith I, et al. Who will be eligible? An investigation of the dementia population eligible for Cholinesterase treatment following the change in NICE guidance. Int Geriatr Psychiatry. 2010;25(7):719-724.

44. Latter, Sue. Safety \& quality in independent prescribing: an evidence review. Nurse Prescribing. 2008;6(2):59-66. 